\title{
The Lysosomal Storage Diseases: A Promising Axis for COVID-19 Future Therapies
}

\author{
Naima Fdil ${ }^{1 *}$, Miloud hammoud ${ }^{1}$, Es-said Sabir ${ }^{1}, K^{2}$ arima Lafha', Abdellatif Laamani², Sara Ali- \\ bou $^{2}$, Wadii Ouahmane ${ }^{2}$, Wafaa Fadili² and Inass Laouad ${ }^{2}$ \\ ${ }^{1}$ Metabolic platform, Cadi Ayad University, Morocco \\ ${ }^{2}$ Department of Nephrology Hemodialysis and renal Transplantation, Mohamed VI University Hospital, Morocco
}

*Corresponding author: Naima Fdil, Metabolic platform, Biochemistry Laboratory, Faculty of Medicine, Cadi Ayad University, Sidi Abbad, BP 40000, Marrakech, Marocco

To Cite This Article: Naima Fdil, Miloud hammoud, Es-said Sabir, Karima Lafha, The Lysosomal Storage Diseases: A Promising Axis for COVID-19 Future Therapies. 2020 - 10(6). AJBSR.MS.ID.001576. DOI: 10.34297/AJBSR.2020.10.001576.

Received: 眥 November 10,2020; Published: 棒 November 19, 2020

Keywords: COVID-19; Lysosomal storage disorders; Fabry disease; Glycosphingolipid

\section{Introduction}

Severe acute respiratory syndrome coronavirus 2 (SARSCoV-2) is the causative agent of the current COVID-19 pandemic; it has been officially named) by the WHO in March 2020 [1]. The current coronavirus pandemic is characterized by high contagion and infection rates and higher mortality among patients with some underlying medical conditions, including those with diabetes, high blood pressure, autoimmune diseases... Caring for high-risk populations is an essential part of managing any communicable disease threat. Patients with inherited metabolic diseases (IMD) are known to be disproportionately affected by respiratory diseases and recurrent infections often resulting in increased morbidity and mortality. IMD, like lysosomal storage diseases (LSD), were included in the group of conditions considered to have an extremely high risk of severe COVID-19 illness [2]. Respiratory symptoms are frequent in patients with LSD with pathomechanisms such as infiltration of pulmonary structures by abnormal cells in Niemann-Pick (NPD) and Gaucher diseases (GD), airway soft tissue infiltration, accompanied by limited chest mobility in mucchopolysaccharidoses diseases (MPSs), dyspnea and wheezing, with a compromised respiratory function by cardiac involvement in Fabry disease (FD) [3]. The hepatosplenomegaly and spinal deformity reduce lung volume in this diseases, it is obvious that a COVID-19 infection will be fa tal for these patients by accelerating respiratory decline. However, few works had addressed this point and few recommendations and practical considerations have been issued by practitioners and experts involved with for the management of this health crisis.

Faced with the rapid spread of the Covid-19, hospitals and referral centers have adopted individual and relative plans to protect, as far as possible, patients with LSD and prevent their infection.

In Morocco, in a cohort of approximately 400 confirmed patients suffering from MPS, GD and FD and of whom only less than 20 percent benefit from regular specific treatment by enzyme replacement therapy, only a single patient with FD had laboratoryconfirmed COVID-19, following a screening for SARS-CoV-2 in hemodialysis patients in our hospital dialysis center where 4 patients died because of the virus.

The patient, a 21-year-old male, was first hospitalized at age of 13 for hyperhidrosis and skin lesions even in the still evoking angiokeratomas; ophthalmologic examination revealed corneal opacity and polar cataract, urea was $1.94 \mathrm{~g} / \mathrm{l}$ and creatinine $58 \mathrm{mg} / \mathrm{l}$. Ultrasound underlined hepatosplenomegaly and a hydronefrosis on the left side These findings were consistent with a diagnosis 
of Fabry disease. The absence of a national reference laboratory at the time did not allow for biochemical or genetic confirmation of the disease. Over the next seven years his renal function deteriorated and he required hemodialysis. Before scheduling him for his first hemodialysis session, urine and blood samples were sent to our laboratory, a thin layer chromatography of the lipid extract from urine revealed the presence of spots corresponding to globotriaosylceramide and the $\alpha$-Galactosidase A activity assayed using dried blood spot was totally deficient. Because of the lack of access by the patient to specific but costly FD therapy, he continued to receive standard nonspecific therapy for the various manifestations of FD including severe anemia, renal failure, peritoneal effusion, marked loss of weight, corneal dystrophy, dry cough...

The patient, despite his critical condition, was asymptomatic to SARS-CoV-2 infection, and was managed by quarantine for 14 days at home. Recent studies have come up with very likely proposals to explain the low rate of LSD patients infected with COVID-19. Indeed, Zimran et al. [4] have speculated that the accumulated glycosphingolipids in patients with GD promote mainly immune tolerance rather than inflammation when exposed to COVID-19. Balout et al. [5] proposed that Niemann-Pick disease type C (NPC) is a lysosomal storage disorder whose consequences result in an "unfavorable" host cell environment that can interfere with the spread of COVID 19.

Such hypotheses can partially explain the almost absent infection of our LSD patients by the virus, even in those who have not benefited from specific ERT treatments. The patient case discussed above suggests that it is possible that other patients in our cohort were infected but they were not detected since they were asymptomatic. Lysosomal diseases seem to preserve patients from infection and / or severe infection with the virus and possibly prevent its spread. Several factors can intervene individually or in combination to explain the low rate infection of patients with LSD. One of the greatest challenges for the virus, along with the immune system, is cell entry. For coronaviruses, cell entry involves two principal steps, receptor binding and membrane fusion; the latter requires activation by host proteases, particularly lysosomal proteases. If we assume that the role of lysosomal proteases is partially or totally disrupted due to metabolites overload in LSDs, this may explain the low infection rate overall in all LSD patients including MPSs. The immune system can also intervene, indeed a consequence of any disease with accumulating metabolites could be that the metabolite-specific-mediated immunity becomes different and may induce new immune activation involving Natural Killer cells and other immune cells, such as B cells [6]. Hence, compared to healthy people, our LSD patients have an "improved " immunity stimulated by overloaded metabolites.

If we focus only on our patient with $\mathrm{FD}$, to explain his asymptomatic infection we could adopt all the hypothesis set out above; as we could also justify it by the role of the gut microbiota in influencing SARS-CoV-2 disease that has been recently well articulated [7] and the fact that the globotriaosylsphingosine, the accumulated glycosphingolipid resulting from $\alpha$-galactosidase deficiency, modifies the biology of gut bacteria, favoring the production of biofilms and altering the composition and shortchain fatty-acid profile of the gut microbiota [8].

We suggest that the infectivity observed in patients with LSD and all hypotheses proposed to explain it, could be used to identify existing and future therapies for COVID19.

\section{Acknowledgment}

The authors acknowledge Doctor Francisco J. Del Castillo for reviewing the scientific content of this manuscript.

\section{References}

1. Aylward BL (2019) Report of the WHO-China Joint Mission on Coronavirus Disease 2019 (COVID-19). The WHO-China Joint Mission on Coronavirus Disease 2019: 16-24.

2. Neufeld EF (1991) Lysosomal storage diseases. Annu Rev Biochem 60: 257-280.

3. Magage S, Lubanda JC, Susa Z, Bultas J, Karetova D, et al. (2007) Natural history of the respiratory involvement in Anderson-Fabry disease. J Inherit Metab Dis 30(5): 790.

4. Zimran A, Szer J, Revel-Vilk S (2020) Impact of Gaucher disease on COVID-19. Intern Med J 50(7): 894.

5. Ballout RA, Sviridov D, Bukrinsky MI, Remaley AT (2020) The lysosome: A potential juncture between SARS-CoV-2 infectivity and Niemann-Pick disease type C, with therapeutic implications. FASEB J 34(6): 7253-7264.

6. Van Eijk M, Ferraz MJ, Boot RG, Aerts JMFG (2020) Lysoglycosphingolipids: presence and consequences. Essays Biochem 64(3): 565-578.

7. Dhar D, Mohanty A (2020) Gut microbiota and Covid-19-possible link and implications. Virus Res 285: 198018.

8. Aguilera-Correa JJ, Madrazo-Clemente P, del Carmen Martínez-Cuesta M, Peláez C, Ortiz A, et al. (2019) Lyso-Gb3 modulates the gut microbiota and decreases butyrate production. Sci Rep 9(1): 1-10. 